\title{
A Review Prevention of Preterm Births and its Complications - An Update
}

\author{
Kulvinder Kochar Kaur ${ }^{1 *}$, Gautam Allahbadia ${ }^{2}$ and Mandeep Singh ${ }^{3}$ \\ ${ }^{1}$ Scientific Director, Dr Kulvinder Kaur Centre for Human Reproduction, Jalandhar, Punjab, India \\ ${ }^{2}$ Scientific Director, Rotunda-A Centre for Human Reproduction, Mumbai, India \\ ${ }^{3}$ Consultant Neurologist, Swami Satyan and Hospital, Jalandhar, Punjab, India \\ *Corresponding Author: Kulvinder Kochar Kaur, Scientific Director, Dr Kulvinder Kaur Centre for Human Reproduction, Jalandhar, \\ Punjab, India.
}

Received: October 11, 2019; Published: November 07, 2019

DOI: 10.31080/ASPE.2019.02.0181

\begin{abstract}
Preterm birth (PTB) is the birth of a baby before 37 completed weeks of pregnancy. Complications of PTB are the biggest reasons of neonatal deaths, causing $35 \%$ of the worlds 3.1 million deaths /year. It has multiple short term in addition to long term effects like cerebral palsy, mental retardation, visual and hearing impairments, learning problems, poor health and slow growth. This review we used a pubmed search engine to identify articles up to 2019 September for identifying articles relevant to PTB and methods used in its prevention. Of the over 65,00 articles we selected 39 articles for this review. Here we have emphasized on role of tocolytics, atosiban, mag sulfate, nifedipine indications and when and when not to use corticosteroids. Further other methods to be used to prevent complications associated with PTB are discussed in detail.
\end{abstract}

Keywords: PTB; Cerclage; Oligodendrocyte Progenitors; Chronic Lung Disease

\section{Introduction}

Preterm birth (PTB) is the birth of a baby before 37 completed weeks of pregnancy [1]. Complications of PTB are the biggest reasons of neonatal deaths, causing $35 \%$ of the worlds 3.1 million deaths/yr [2]. It has multiple short along with long term effects like cerebral palsy, mental retardation, visual and hearing impairments, learning problems, bad health and low growth [3].

Globally as per WHO rates of preterm births point that of the 135 million live births in 2010, 15 million babies were born much early, giving a rate of PTB rate of $11.1 \%$ and $>60 \%$ of PTB s took place in sub-Saharan Africa and South Asia [4].

\section{Methods}

Thus we carried out a systematic review where we used the pubmed search engine using the MeSH terms like Preterm birth; congenital malformation and PTB; prevention of PTB; Prevention of side effect like chronic lung disease; central nervous system damage prevention; indications of caesarean section versus vaginal delivery; role of various drugs like progesterone, magnesium sulphate: atosiban, tocolytics, antibiotics for mother.

\section{Results and Discussion}

We found a total of 62,508 articles of which we selected 39 articles for this review. No meta-analysis was done.

PTB in Ethiopia

Approx. 350, 000 children <5yrs die yearly secondary to PTB complications in Africa that includes Ethiopia. Maximum rates of perinatal mortality are in Western Africa in PTB, especially in countries like Sierra, Lone and Liberia, that are getting destroyed by Ebola [5].

Prior studies, earlier history of PTB was the biggest predictor of chances of repeat PTB and if these risk factors are not found properly and controlled, they =>enhanced incidence of PTB. Hence 
identifying and referring for proper examination of these women in early pregnancy is essential to reduce the results of PTB [6].

Developing countries such as Ethiopia don't have data that can be relied on regarding PTB has not been examined properly.

Thus, Aregawi., et al. tried to find out the prevalence and associated factors of PTB among mothers delivering in Axum and Adwa public hospitals, Tigray, North Ethiopia in 2018. Their study revealed that $13.3 \%$ of total 472 mothers had a preterm delivery. Since it was a rural delivery (AOR=2.13,95\%CI1.07, 4.22), short interpregnancy interval (AOR=5.4,95\% CI $(1.32,22.05)$, previous preterm birth $(\mathrm{AOR}=3.74,95 \% \mathrm{CI}(1.03,16.34)$ Premature rupture of membranes (AOR=4.14, 95\%CI1.92, 8.89), induced onset of labor $(\mathrm{AOR}=2.49 .95 \% \mathrm{CI}(1.05,5.85)$ multiple pregnancy $(\mathrm{AOR}=5$. $69,95 \%$ CI $(2.27,14.28)$, malaria during pregnancy $(\mathrm{AOR}=4,71$, 95\%CI $(1.98,11.23)$ Presence of chronic illness (AOR=4.55, 95\%CI $(1.83,11.26)$ had a significant association with PTB [7].

Role of smoking

PTB is a significant global problem and the commonest cause of mortality of newborns. Tobacco smoking has been related with PTB for $>50 y r s$. It is not clear what are the modes by which smoking has its effects on pregnancy. Hence Ion and Bermal discussed rates of prematurity and smoking in pregnancy. The process and biochemical pathways that they posited by which this interaction gets mediated. The implicated mechanisms are nicotine induced vasoconstriction, carbon monoxide stimulated fetal hypoxia, cadmium interference with calcium signaling, changed steroid hormone synthesis, interference with prostaglandin production, besides different responses to oxytocin. Relative importance of each pathway has not been found as yet. Future work is essential to find the modes via which smoking influences gestation length along with the parturition process. Further the risks of nicotine replacement in pregnancy needs to be probed further [8].

Following decades of increasing PTB rates in USA and other countries, recent prematurity rates seem to be reducing. In spite of this optimistic pattern PTB remains>in USA, where nearly 1/8 infants gets born early, as compared to other developed countries. Prevention of PTB is considered a public health priority in view of the probability of decreasing infant and childhood morbidity and mortality associated with this condition. Progress has not been much unfortunately. Biggest challenges regarding studying outcome is that PTB is a complex condition that resulted from multiple etiologic pathways. Experts have developed novel frameworks for classification and studying PTB based on phenotype. These classification systems proposed have only recently been adopted, but a separate perspective on a longstanding problem which has the potential to $=>$ new discoveries [9].

\section{Role of CLD in PTB}

Very low birth weight (VLBW) infants born prior to $33 \mathrm{wks}$ of pregnancy at Boston Medical Center (BMC) had a >chances of chronic lung disease (CLD), averaging 35\% from 2006-2012. Marked work done to decrease the incidence was not successful.

CLD is a developmental disease with multiple aetiologies, although reduced mechanical ventilation (MV) might decrease the incidence of CLD. Avery., et al. [10] documented that Columbia University in New York possessed the minimal incidence of CLD in contrast to 7 other main medical centres. This was thought to be in view of early intervention utilizing bubble continuous positive air way pressure (bCPAP) that avoided MV [10]. This was confirmed by Marter in 2000 [11]. Subsequently 3RCTs [12] and various cohort and observational research [13] which approved this method. In all it was shown that a decrease in MV, and 3 documented marked decrease in CLD $[13,14]$.

A multidisciplinary team present at St Elizabeth's Medical Center (SEMC) in Boston, initiated a lot of respiratory practices, on the basis of the Columbia approach, that included the use of bCPAP only, along with availability of bCPAP in the DR, strict criteria for intubation and extubation along with continued bCPAP for avoidance of supplemented oxygen $\left(\mathrm{O}_{2}\right)$ and help in lung growth [14], in infants both at younger than 33 wks gestational age (GA) without side effects. The incidence of CLD reduced by 53\%. Thus Levesque., et al. posited that they could decrease the incidence of CLD in BMC utilizing the same approach. But marked differences between the neonatal intensive care units (NICU) at SEMC in contrast with those at BMC was there which increased doubt about the results. Their multidisciplinary task force used 6 plan-do-study compared to cycles for examining for testing their interventions. The initial result was the quarterly percentage of infants diagnosed with CLD, other outcomes were the percentage of infants earlier managed with CPAP, intubation $<72 \mathrm{~h}$ of age, utilization of a nasal cannula, 
and number of days of ventilation, $\mathrm{O}_{2}$ and/or CPAP, process ways used prematurely. In the 55 infants born before and 75 born following the task force interventions had similar demographics, other than GA, that was lower before. A reduction in CLD by $55.5 \%$ (from $37.5 \%$ to $16.7 \%$ ) occurred. Quarterly percentage of infants needing intubation reduced from $87.5 \%$ to $40.6 \%$. Quarterly approximate days of ventilation reduced from 11.2 to 6.1 and number of days of supplemental $\mathrm{O}_{2}$ reduced from 44.1 to 25.4. although use of CPAP rose. No difference in adverse effects like mortality, pneumothorax, utilization of postnatal steroids, or any retinopathy of prematurity. Incidence of patent ductus arteriosus (PDA) reduced from $60 \%$ to $33 \%(\mathrm{p}<0.01)$. Thus concluding that they decreased the incidence of CLD among their very low birth weight infants $<33$ wks GA by over $50 \%$ without enhancing any measured adverse outcomes. The incidence of PDA reduced [15].

\section{Committee opinion for PTB prevention in France}

PTB, in France involves 60, 000 neonates/year (7.4\%) of which $50 \%$ get delivered following spontaneous onset of labor. Of the preventable risk factors of spontaneous prematurity, only stopping smoking, correlates with reduction in prematurity (level of evidence [LE] 1). Thus this is recommended (grade A). Screening and treating vaginal bacterial excess in general population is not recommended (grade A). Women who are asymptomatic with no history of preterm delivery and presence of a short cervix between 16 and 24 weeks is the only population that needs vaginal progesterone and thus recommended (Grade B). On the basis of a history indicated cerclage is not recommended if only past history of conization (grade C), Uterine malformation (professional consensus), isolated history of preterm delivery (grade $B$ ) or twin pregnancies in primary (grade B), or secondary (grade C) prevention of PTB. A history indicated cerclage gets recommended for single pregnancy with a history of a minimum 3 late miscarriages or preterm deliveries (grade A). When past history of single pregnancy delivery prior to $34 \mathrm{wks}$ GA, Ultrasound cervical length screening is recommended between 16 and 22 weeks GA for being able to propose a cerclage if length $<25 \mathrm{~mm}$ before $24 \mathrm{GA}$ (Grade C). Cervical pessary should not be inserted to prevent PTB in a general population of asymptomatic women having a twin pregnancy (grade A) and in population of asymptomatic women with a short cervix ( (professional consensus). Though using universal transvaginal cervical length measurement at $18-24 \mathrm{wk}$ of GA in women with singleton pregnancy and no history of PTB can be used by individual doctors, this screening can't be recommended universally. If already pre- term labor initiated i). It cant be recommended one of the methods over another (Ultrasound of the cervical length, vaginal examination, fetal fibronectin) to predict PTB (Grade B); ii). Routine antibiotic therapy is not recommended (grade A) iii). Prolonged hospitalization (grade B) and bed rest (grade $\mathrm{C}$ ) is not recommended. In contrast to placebo, tocolytics are not related to a decrease in perinatal mortality or morbidity (LE2) and maternal severe adverse effects might occur with tocolytics (LE4). Atosiban and nifedipine (grade B) in contrast to beta mimetics (grade C) can be used for tocolysis in spontaneous preterm labor without PROM. Maintenance tocolysis is not recommended (grade B). Antenatal corticosteroids administration is recommended every woman at risk of preterm delivery before 34wks GA (Grade A). Following 34wks, evidence are not consistent enough to recommend systemic Antenatal corticosteroids treatment (grade B), however a course might be indicated in the clinical situations related with > risk of severe respiratory distress syndrome, mainly incase of planned caesarean delivery ( (grade C). Repeated courses of Antenatal corticosteroids are not recommended). (Grade A). Rescue courses are not recommended (professional consensus). Magnesium sulfate administration is recommended to women at high risk of imminent PTB before 32wkGA (Grade A). LSCS is not recommended in case of vertex presentation (professional consensus). A delayed cord clamping might be considered if the neonatal state so permits (professional consensus), Thus concluding that for Antenatal corticosteroids and Magnesium sulfate administration, diagnostic tools or prenatal pharmacologic treatments implemented since 30yrs to prevent PTB and its consequences have not matched expectations of caregivers and families [16].

\section{Improving quality of care for PTB along with outcome}

For the prioritization, this included people affected by PTB and health care practitioners In the UK relevant to PTB. The qualitative work on PTB and the development of a questionnaire involved parents of infants born at 3 maternity hospitals in southern England. The medical device was developed at Liverpool Women's Hospital. The survey of practice involved UK neonatal units. The randomized trial was conducted at 8 UK tertiary maternity hospitals. For prioritization, 26 organizations and 386 individuals; for the interviews and questionnaire tool, 32 mothers and seven fathers who had a baby born before 32 weeks' gestation for interviews evaluating the trolley, 30 people who had experienced it being used at the birth of their baby (19 mothers, 10 partners and 1 grandmother) and 20 clinicians who were present when it was being used; for the trial, 
261 women expected to have a live birth before 32 weeks' gestation, and their 276 babies. Providing neonatal care at very preterm birth beside the mother, and with the umbilical cord intact; timing of cord clamping at very preterm birth were the interventions needed. The main outcome measures utilized were Research priorities for preterm birth; feasibility and acceptability of the trolley; feasibility of a randomised trial, death and intraventricular haemorrhage. Systematic review of Cochrane reviews (umbrella review); framework synthesis of ethics aspects of consent, with conceptual framework to inform selection criteria for empirical and analytical studies. The comparative review included studies using a questionnaire to assess satisfaction with care during childbirth, and provided psychometric information were the review methods utilized. Thus Duley., et al. prioritization suggested 104 research topics for preterm birth, with the main 30 arranged according to ranking. An ethnographic analysis of decision-making during this process suggested ways that it might be improved. Qualitative interviews with parents about their experiences of very preterm birth identified two differences with term births: the importance of the staff appearing calm and of staff taking control. Following a comparative review, this led to the development of a questionnaire to assess parents' views of care during very preterm birth. A systematic overview summarised evidence for delivery room neonatal care and revealed significant evidence gaps. The framework synthesis explored ethics issues in consent for trials involving sick or preterm infants, concluding that no existing process is ideal and identifying three important gaps. This led to the development of a two-stage consent pathway (oral assent followed by written consent), subsequently evaluated in their randomised trial. Their survey of practice for care at the time of birth showed variation in approaches to cord clamping, and that no hospitals were providing neonatal care with the cord intact. They showed that neonatal care could be provided beside the mother using either the mobile neonatal resuscitation trolley they developed or existing equipment. Qualitative interviews suggested that neonatal care beside the mother is valued by parents and acceptable to clinicians. Their pilot randomised trial compared cord clamping after 2 minutes and initial neonatal care, if needed, with the cord intact, with clamping within 20 seconds and initial neonatal care after clamping. This study demonstrated feasibility of a large UK randomised trial. Of 135 infants allocated to cord clamping $\geq 2$ minutes, 7 (5.2\%) died and, of 135 allocated to cord clamping $\leq 20$ seconds, 15 (11.1\%) died (risk difference $-5.9 \%$, 95\% confidence interval $-12.4 \%$ to $0.6 \%)$. Of live births, 43 out of 134 (32\%) allocated to cord clamp- ing $\geq 2$ minutes had intraventricular haemorrhage compared with 47 out of $132(36 \%)$ allocated to cord clamping $\leq 20$ seconds (risk difference -3 . $5 \%, 95 \% \mathrm{CI}-14.9 \%$ to $7.8 \%$ ). Limitations of the study being the small sample for qualitative interviews about PTB, and it being a single centre evaluation of neonatal care, other than the mother and it being a pilot trial. Thus they concluded that their research helps in getting insight into what parents experience at the time of PTB, besides giving informed clinical guidelines, along with the research agenda. Their 2 stage consent method is recommended for research during the intrapartum clinical trials. Further their pilot trial will add to the single person data metaanalysis, whose results will help in designing next trials. Further research in preterm birth should take account of the top priorities. Further evaluation of neonatal care beside the mother is merited, and future trial of alternative policies for management of cord clamping should take account of the meta-analysis. This study was registered as PROSPERO CRD42012003038 and CRD42013004405. In addition, Current Controlled Trials ISRCTN21456601 [17,19].

For comparison of the incidence of post-PDA ligation syndrome after surgical vs percutaneous closure of PDAs in very low birth weight (VLBW) infants, Serrano., et al. studied a cohort of 50 infants undergoing surgical ligation with 25 infants undergoing percutaneous closure. Comparisons between groups were done using 2 sample $t$ tests and a Fisher's exact test. Comparison of pre vs post variables within each group were made using paired tests. Patients who underwent surgical ligation had a >requirement for initiation of post procedure hemodynamic support (12/59vs $0 / 24$, $\mathrm{p}=0.16)$, a >post procedure peak $\mathrm{FiO}_{2}$ (0.64 vs $0.43, \mathrm{p}=0.004$ ) and a bigger absolute alteration in peak $\mathrm{FiO}_{2}$ ( 0.23 vs $\left.0.09, \mathrm{p}=0.008\right)$. Thus concluding that VLBW infants having percutaneous closure of their PDA did not experience post ligation syndrome and had less escalation of respiratory support in contrast to those undergoing surgical ligation $[17,19]$.

Neuroprotection with grafter Oligodendrocyte progenitor in perinatal excitotoxicity

The CNS communicates through transmission of electrical signals. Speed of these signals is necessary for normal motor, sensory and integrative functions. For this the myelin membrane is a necessary component underlying this process, by insulating axons and permitting rapid transmission of these signals [18]. Oligodendrocytes (OLs) are the cells that produce and maintain myelin in the brain. In the adult CNS, damage to or loss of neurons, axons and 
OLs $=>$ demyelination that cause impaired neurological functions and disability [19]. The harmful effects of myelin loss are seen in a lot of inherited along with acquired myelin disorders [20duncanradcliffe 16]. Mutations in myelin genes, called leuko dystrophies, have been implied in various developmental human diseases like Pelizaeus -Merzbacher disease [21,22]. One in 8 babies is born before term (37-40wks), and $1.44 \%$ of babies $(56,000 / \mathrm{yr})$ are born with a birth wt of $1250 \mathrm{gm}$ or< [23].

2 biggest reasons of neonatal brain injury are extreme prematurity and hypoxic ischemic encephalopathy. In USA ( $[21,22]$ one in 8 babies is born before term (37-40wks), and $1.44 \%$ of babies $(56,000 / \mathrm{yr})$ are born with a birth wt of $1250 \mathrm{gm}$ or $<$ [23]. These small preterm babies are at high risk of death or neurodevelopmental impairment, including cerebral palsy [21,22]. In USA, care of preterm infants represents $>50 \%$ of paediatric health expenses. Preterm infants represent a population of that is vulnerable to white matter (WM) loss, and the size of this population is increasing continuously. Advances in perinatal care have resulted in marked improvement in the survival of very premature $(<30 \mathrm{wk}$ GA) infants [24]. In particular developed countries data of the PTB rate has increased remarkably in the last 2 decades, with continuation of increase. In 2005 there were roughly 12.9 million PTB globally with roughly $90 \%$ of them surviving past infancy [25]. Of the survivors up to $1 / 4^{\text {th }}$ develop cerebral palsy, and $25-50 \%$ have cognitive disabilities and developmental deficits. These impairments are mainly in view of the developmental state of the preterm infants cerebral WM. Utilizing a myelin deficient rat Ikeda., et al. earlier demonstrated that OLPs grafted into their brains migrate, integrate and reach naked axons to myelinate them [26,27]. Other workers have also utilized mutant rodents markedly for the same reason [28]. These mutant models have helped in the examination of graft performance along with host graft interaction in a noninflammatory microenvironment. Conversely transplantation of healthy OLPs into hostile microenvironment (like inflammation in the host brain) still pose challenges.

Since the cerebral WM is getting formed in preterm infants, under poor cerebral blood flow, it is vulnerable to hypoxic ischaemia. This causes periventricular leukomalacia, a common and severe form of cerebral WM injury (WMI) [29]. hypoxic ischaemia $=>$ greater level of extracellular glutamate, that in turn causes free reactive oxygen species (ROS). Pre-OLs are vulnerable to free radical insult, ending in their death [30]. The number of glutamate N-methyl -D-Aspartate (NMDA) receptors are involved in WMI in addition to increased levels of extracellular glutamate that might be caused by inadequate glutamate transporters and glutamate induced excitotoxicity have been produced with the use of NMDA injections [31]. Activation of NMDA receptors, escalates intracellular calcium, proapoptotic pathways through caspase3 activation, free radical generation, and lipid peroxidation, causing marked and widespread injury to the developing brain [22].

Right now there is no cure for WMI hence affected persons will undergo lifelong damaging effects of myelin loss that includes ventriculomegaly along with impaired neurological function. Two promising treatment approaches are i) OLP transplants to provide the brain with healthy OLPs for myelination. Earlier works have tried to graft OLPs glutamate excitotoxicity (GME) [32]. Grafted cells are known to survive in comparable numbers to those in control mice several weeks post grafting. For the grafted OLPs survival and migration was markedly decreased, but behavioral scores, myelination and neuropathological outcomes were improved, most probably due to early trophic factors after GME. Like in a mouse model of excitotoxicity, the authors have demonstrated that TSC1 (a combination of transferring and insulin growth factor 1 (IGF1)) stimulates both, endogenous neural stem cells and OLP lineage progression and maturation. TSC promoted enhanced OL proliferation and migration in the corpus callosum (CC) and striatum, and then regenerating myelin and reducing ventricular size [33,34]. In this study, Hirose- Ikeda., et al. considering cell replacement and integration as therapeutic goals, they evaluated if OL progenitors (OLPs) grafted into the brain parenchyma of mice that had been subjected to an excitotoxic insult might rescue WM injury. To that end they utilized a well established model of glutamate excitotoxic injury. 4 day old mice got a single intraparenchymal injection of the glutamate receptor antagonist NMDA alone or along with TSC1 in the presence or absence of OLPs grafted into the brain parenchyma. Energetics and expression of stress proteins and OL developmental specific markers were evaluated. Comparing the proteomic profile/ treatment was also checked. They observed that OLPs did not survive in the excitotoxic environment on grafting alone. On the other hand, on combining with TSC1 survival and integration of grafted OLPs survival and integration of grafted OLPs was seen. Moreover energy metabolism in OLPs was markedly enhanced by NMDA and modulated by TSC1. The proteomic profile following the vari- 
ous treatments showed increased ubiquitination and stress/heat shock protein 90 in response to NMDA. These changes got reversed in the presence of TSC1 and ubiquitination got reduced. These results got in this preclinical study suggest that using combinatorial intervention that include both trophic support and healthy OLPs constitute a promising method for long term survival and successful graft integration. Thus they established right conditioning of the host brain environment for promoting long term survival along with integration of grafter OLPs into an inflamed neonate host brain. Experimental procedures were done under the US Public Health Service Guide for the Care and use of laboratory animals, approved by Institutional Animal Care Committee [35].

\section{PTB and congenital birth defects}

Prenatal myelomeningocele (MMC) closure has been done in US for over 2 decades. Earlier work has concentrated on clinical results of prenatal MMC closure, it has not been known, what the cost of this surgery vs that of postnatal MMC Closure with regard to both child along with mother at 1 year. Thus Kessler., et al. retrospectively analysed a prospective database of patients who underwent prenatal and postnatal MMC Closure between 2011 and 2018 with 1 year follow up. Charge data for the admissions relevant to this were converted to cost estimate using the authors institution 's Medicare hospital-specific cost to charge ratio. Children, mothers, and mother/child pairs were taken separately. The primary result was cost. Secondary outcomes were the requirement of hydrocephalaus treatment, length of stay (LOS), and readmissions. Other covariates taken into account were GA at birth, MMC lesion level and obstetric complications. The median cost of care for children in the prenatal group was $>$, although not significantly so, at $\$ 58$, 406.71 (IQR $\$ 16,900.24-\$ 88,951.01$ ) compared with $\$ 49,889.95$ (IQR $\$ 38,425.18-\$ 115,163.86)$ for children in the postnatal group $(\mathrm{p}=0.204)$. The median cost for mothers in prenatal group was significantly > at $\$ 24,548.29$ (IQR $\$ 20,231$. 565-\$36, 862.31) in contrast to $\$ 5087.30(\$ 4430.72-5362.56)(\mathrm{p}<0.001)$. The median cost for the mother/child pairs in the prenatal group was $\$ 102,377.75$ (IQR \$37, 384.30-\$118, 527.74) in comparison with $\$ 55,667.82$ (IQR \$42, 840.78-\$120, 058.06) ( $\mathrm{P}=0.45)$. Children in the prenatal group had a lower GA at birth (235.81 days vs 265.77 days, $\mathrm{p}<0.001)$, and fewer readmissions $(33.3 \%$ vs $72.7 \%, \mathrm{p}<0.001)$, and hydrocephalaus treatment was < common $(33.3 \%$ vs $90.9 \%$, $\mathrm{p}<0.001)$. Index LOS did not differ between children in the prenatal and postnatal group (26.8 days vs 23,5 days, $p=0.63$ ). Mothers in the prenatal group had longer LOS (15.92 days vs 4.68 days, $\mathrm{p}<0.001)$ and $>$ readmissions $(18.5 \%$ vs $0.0 \%, \mathrm{p}=0.06)$. Thus concluding that the cost of prenatal vs postnatal MMC Closure did not significantly differ from a hospital perspective at 1 year although variability of cost was high in both groups. If mother considered alone, prenatal MMC Closure was costlier. Further work is required to find cost from a patient along with societal perspective both at 1 year and beyond [36].

Serrano., et al. tried to compare the incidence of post patent ductus arteriosus (PDA) ligation syndrome after surgical vs percutaneous closure of PDAs in very low birth weight (VLBW) infants. They conducted a cohort study in which comparison of 59 infants who underwent surgical ligation while 25 infants underwent percutaneous closure. Comparisons of pre vs post variables within each group were done using paired t-tests. Pts who underwent surgical ligation had a higher requirement for initiating post procedure hemodynamic support $(12 / 59$ vs $0 / 24, \mathrm{p}=0.016$, a higher post procedure peak FiO2 ( 0.64 vs $0.43, p=0.004$ ), and a large absolute change in peak FiO2 ( 0.3 vs $0.09, \mathrm{p}=0.008$ ). Thus concluding that VLBW infants who had percutaneous closure of their PDA did not experience post ligation syndrome and had less enhancement of respiratory support as compared with infants who underwent surgical ligation [37].

\section{Role in retinopathy in $\mathrm{PTB}$}

Petrachkova., et al. tried to develop a mathematical model to predict the development of threshold stages (type 1) of active retinopathy of prematurity (ROP) based on neonatal risk factors in premature infants. 69 premature infants from a perinatal center with GA of 22-31 wks and birth weight of 499-1500grams. Patients were divided into 2 groups depending on the type of ROP: the main group-40 infants with a threshold stage (type 1) of ROP, needing laser treatment; the control group-29 children with spontaneous regression of the initial (1-2) stages (type 2) of the disease. A model for predicting the development of ROP threshold stage was builtusing mathematical justification. This study established potential predictors of type 1. ROP: staying in the intensive care unit for more than 30days ( $\mathrm{p}=0.011$ ), duration of child's staying on mechanical ventilator $>$ than 30 days $(\mathrm{p}=0.002)$ : parenteral nutrition for $>13$ days $(p=0.013)$, presence of respiratory distress syndrome of 3 rd degree $(\mathrm{p}<0.001)$; presence of bronchopulmonary dysplasia $(p=0.034)$. These predictors were used to design a mathematical model for predicting the development of threshold stage of ROP. Thus concluding that the proposed prognostic model of type 1 ac- 
tive ROP is characterized by high information content and can be recommended for use in clinical practice of neonatal medical institutions [38].

Role in autoimmune rheumatic disease

For evaluating the PTB phenotypes in women with different autoimmune rheumatic diseases in a large population based cohort Kolstad., et al. conducted a retrospective study where all singleton births in California between 2007-2011 were examined. Pts with autoimmune disease at delivery were isolated by ICD 9 codes for systemiclupus erythematosus (SLE), Systemic sclerosis (SSc), rheumatoid arthritis (RA), polymyositis/dermatomyositis (DM/PM), and juvenile idiopathic arthritis (JIA). Maternally linked hospital and birth certificate records of 2,481,516 deliveries were assessed (SLE $n=2,272$, RA $n=1,501$, SSc $n=88$, JIA $n=187$, $\mathrm{D}, / \mathrm{PM} \mathrm{n}=38$ ) Multivariable Poisson regression models estimated risk ratios (RRs) for different PTB phenotypes (relative to term deliveries) for each autoimmune disease compared to the general obstetric population adjusting for maternal age, race/ethnicity, BMI, smoking, education, payer, parity, and prenatal care. PTB was assessed overall (20-36wks) and by subphenotypes: preterm premature rupture of menbranes (PPROM), spontaneous, or medically indicated PTB. Risk of PTB-overall and each phenotype was partitioned by GA: early (20-31wks) and late (32-36wks). Risks of PTB were increased for each autoimmune disease examined (SLE (RR3.27 95\%CI 3.01-3. 56), RA (RR 2.0495\%CI 1.79-2.33), SSc (RR3. 74 95\%CI 2.51-5.58), JIA (R2.23 95\%CI 1. 54-3.23) and DM/P) RR 5.2695\% CI3.128.89). These increased risks were observed for most of the phenotypes as well. Thus women with symptomatic autoimmune diseases appar to have an increased risk of several PTB phenotypes. Thus, preconception counseling and close monitoring during pregnancy is critical [39].

\section{Conclusions}

Thus in this review we have considered how to prevent PTB, with the committee reports of do's and don'ts in antenatal women at risk for preterm labor including use of atosiban, magsulfate, nifedipine, role of corticosteroids only prior to $34 \mathrm{wks}$, indications of cerclage, tocolytics and type of oxygen therapy to be given to the PTB infant to avoid CLD. Further with white matter of PTB infants not fully formed these children remain at risk of excitotoxic injury with risk of cerebral palsy, mental retardation, hence trials are being done to see if role of oligodendrocyte progenitors may help in preventing this. It was seen that just with NMDA it was not avoided but with addition of TSC's this got avoided. Further cost of repairing congenital malformations like Meningomyelocoele prenatal and postnatal is considered along with repair of PDA and risks of retinopathy and risk of PTB in autoimmune conditions has been discussed.

\section{Bibliography}

1. Lockwood C., et al. "Overview of preterm labor and delivery". UpToDate 19 (2011).

2. Liu L., et al. "Global regional and national causes of child mortality :an updated systematic analysis”. Lancet (2012).

3. WHO (World Health Organization). March of Dimes, the Partnership for Maternal Newborn and Child Health and Save the Children Born Too Soon :the global action report on preterm births, Geneva, World Health Organization (2012).

4. Blencowe H., et al. "National, regional and worldwide estimates of preterm births fates in the year 2010 with time trends for selected countries since 1990: a systematic analysis". Lancet (2012).

5. WHO (and Child Health and Save the Children Health Organization). UNICEF (United Nations Children's Fund) March of Dimes, the Partnership for Maternal Newborn and Child Health and Save the Children. World prematurity day (2013).

6. Offiah I., et al. "Clinical risk factors for preterm birth, preterm birth". Mother and Child (2012).

7. Aregawi G., et al. "Preterm births and associated factors among mothers who gave birth in Axum and Adwa Town public Hospitals, Northern Ethipia 2018". BMC Research Notes 12 (2019): 640.

8. Ion R and Bemal AL. "Smoking and Preterm Birth". Reproductive Sciences 22 (2015): 918-926.

9. Frey HA and Klebanoff MA. "The epidemiology, etiology, and costs of Preterm Birth". Seminars in Fetal and Neonatal Medicine 21 (2016): 68-73.

10. Avery ME., et al. "Is chronic lung disease in low birth weights preventable? A survey of 8 centers". Paediatrics 79 (1987): 2630.

11. Van Marter LJ., et al. "Do clinical markers of barotraumas and oxygen toxicity explain interhospital variation in rates of chronic lung disease? The Neonatology Committee for the Developmental Network". Paediatrics 105 (2000): 1194-1201. 
12. Finer NN., et al. "Early CPAP versus surfactant in extremely preterm infants". The New England Journal of Medicine 362 (2010): 1970-1979.

13. DeKlerk AM and De Klerk KK. "Nasal continuous positive airway pressure and outcomes of infants". Journal of Paediatric Child Health 37 (2001): 161-167.

14. Aly H., et al. "Does the experience with the use of nasal continuous positive airway pressure improves overtime in extremely low birth weight infants?". Paediatrics 114 (2004): 697-702.

15. Levesque BM., et al. "Improving Respiratory Support Practices to Reduce Chronic Lung Disease in Premature Infants". Paediatric Quality and Safety 4.4 (2019): e193.

16. Sentiles L., et al. "Prevention of sontaneous Preterm Birth (excluding Preterm premature rupture of membranes): Guidelines for Clinical Practice-Text of the Guidelines (short text)". Journal of Gynecology Obstetrics and Human Reproduction (Paris) 45 (2016): 1446-1456.

17. Duley L., et al. "Improving quality of care and outcome art very preterm birth: the preterm birth research programme, including the Cord Pilot RCT".

18. Nave KA. "Myelination and the trophic support of long axons". Nature Reviews Neuroscience 11 (2010): 275-283.

19. Blumenthal I. "Periventricular leukomalacia: A review". European Journal of Paediatrics 163 (2004): 435-442.

20. duPlessis AJ and Volpe JJ. "Perinatal brain injury in the preterm and term newborn". Current Opinion on Neurobiology 15 (2002): 151-157.

21. Hamilton BE., et al. "Annual summary of vital statistics 20102011". Paediatrics 131 (2013): 548-558.

22. O'Shea TM., et al. "ELGAN Study Investigators. The ELGAN Study of the brain and the related disorders in extremely low gestational age new-borns". Early Human Development 85 (2009): 719-725.

23. Juul SE and Ferriero DM. "Pharmacologic neuroprotective strategies in neonatal brain injury". Clinical Perinatology 41 (2014): 119-131.

24. Rees $\mathrm{S}$ and Inder T. "Fetal and neonatal origins of altered brain development”. Early Human Development 81 (2005): 753-761.
25. Beck S., et al. "The worldwide incidence of preterm birth: a systematic review of maternal mortality and morbidity". Bulletin of the World Health Organization 88 (2010): 31-38.

26. Espinosa de los Montena A., et al. "Transplantation of cultured promyelinating Oligodendrocytes into normal and myelin deficient rat brain". Developmental Neuroscience 14 (1992): 98104.

27. Espinosa de los Montena A., et al. " Transplantation of CC4 Oligodendrocytes progenitor cells in the myelin deficient rat brain results in myelination of axons and enhanced Oligodendroglial markers". Journal of Neuroscience Research 50 (1997): 872-887.

28. Duncan ID and Radcliffe AB. "Inherited and acquired disorders of myelin: the underlying myelin pathology". Neurology 283 (2016): 452-475.

29. Deng W. "Neurobiology of injury to the developing brain". Nature Reviews Neurology 6 (2010): 328-336.

30. Khwaja o and Volpe JJ. "Pathogenesis of cerebral white matter injury of prematurity". Archives of Disease in Childhood: Fetal and Neonatal 93 (2008): F153-161.

31. Felt BT., et al. "Early appearance of functional deficits after neonatal excitotoxic and hypoxic -ischaemic injury: fragile recovery after development and role of the NMDA receptor". Developmental Neuroscience 24 (2002): 418-425.

32. Porambo M., et al. "Transplanted glial restricted precursor cells improve neurobehavioral and neuropathological outcomes of a mouse model of neuronal white matter injury despite limited cell survival". Glia 63 (2015): 452-465.

33. Espinosa -Jeffrey A., et al. "White matter loss in a mouse model of periventricular leukomalacia is recued by trophic factors". Brain Science 3 (2013a): 1461-82.

34. Espinosa -Jeffrey A., et al. "Efficient generation of Viral and Integration - Free Human Induced Pluripotent Stem Cell-Derived Oligodendrocytes". 38 (2016a): 2D.18.1-2D.18.27.

35. Hirose-Ikeda M., et al. "Trophic factors are essential for the survival of grafted Oligodendrocytes progenitors and for neuroprotection after perinatal excitotoxicity". 15 (2019): 557568.

36. Kessler BA., et al. "Cost of prenatal versus post natal myelomeningocele closure for both mother and child at 1 year of life". Neurosurgery Focus 47 (2019): E15. 
37. Serrano RM., et al. "Comparison of 'post -patent ductus arterial ligation syndrome' in premature infants after surgical ligationvs percutaneous closure". Perinatology (2019).

38. Petachkova MS., et al. "Modern approaches to predicting the development of active type1 retinopathy of prematurity". Vestnik Oftalmologii 135 (2019): 50-59.

39. Kolstad KD., et al. "Preterm birth phenotypes in women with autoimmune rheumatic diseases :A population based cohort study". BJOG (2019).

Volume 2 Issue 12 December 2019

(C) All rights are reserved by Kulvinder Kochar Kaur., et

al. 